\title{
Seizure control after radiosurgery for cerebral arteriovenous malformations: a 25-year experience
}

\author{
Ajay Niranjan, MD, MBA, Ahmed Kashkoush, BS, Hideyuki Kano, MD, PhD, \\ Edward A. Monaco III, MD, PhD, John C. Flickinger, MD, and L. Dade Lunsford, MD \\ Department of Neurological Surgery, University of Pittsburgh, Pennsylvania
}

OBJECTIVE Seizures are the second-most common presenting symptom in patients with lobar arteriovenous malformations (AVMs). However, few studies have assessed the long-term effect of stereotactic radiosurgery (SRS) on seizure control. The authors of this study assess the outcome of SRS for these patients to identify prognostic factors associated with seizure control.

METHODS Patients with AVM who presented with a history of seizure and underwent SRS at the authors' institution between 1987 and 2012 were retrospectively assessed. The total cohort included 155 patients with a mean follow-up of 86 months (range 6-295 months). Primary outcomes assessed were seizure frequency, antiepileptic drug regimen, and seizure freedom for 6 months prior to last follow-up.

RESULTS Seizure-free status was achieved in 108 patients (70\%), with an additional 23 patients (15\%) reporting improved seizure frequency as compared to their pre-SRS status. The median time to seizure-free status was estimated to be 12 months (95\% Cl 0-27 months) as evaluated via Kaplan-Meier survival analysis. The mean seizure frequency prior to SRS was 14.2 (95\% Cl 5.4-23.1) episodes per year. Although not all patients tried, the proportion of patients successfully weaned off all antiepileptic drugs was $18 \%$ (28/155 patients). On multivariate logistic regression, focal impaired awareness seizure type (also known as complex partial seizures) and superficial venous drainage were significantly associated with a decreased odds ratio for seizure-free status at last follow-up (OR 0.37 [95\% $\mathrm{Cl} 0.15-0.92]$ for focal impaired awareness seizures; OR 0.36 [95\% $\mathrm{Cl} 0.16-0.81]$ for superficial venous drainage). The effects of superficial venous drainage on seizure outcome were nonsignificant when excluding patients with $<2$ years of follow-up. AVM obliteration did not correlate with long-term seizure freedom ( $p=0.202$, chi-square test).

CONCLUSIONS This study suggests that SRS improves long-term seizure control and increases the likelihood of being medication free, independently of AVM obliteration. Patients with focal impaired awareness seizures were less likely to obtain long-term seizure relief.

https://thejns.org/doi/abs/10.3171/2018.7.JNS18304

KEYWORDS antiepileptic drugs; prognosis; risk factors; stereotactic radiosurgery; vascular disorders

$\mathrm{S}$ EIZURES are the second-most common presentation of cerebral arteriovenous malformations (AVMs). ${ }^{4}$ AVM-associated seizures are typically focal, with the neuroanatomical location predicting seizure semiology. The prevalence of seizures in untreated AVMs ranges from $11 \%$ to $33 \%$, depending on factors such as male sex, cortical location, superficial drainage, and large nidus volume. . $^{4,11,25}$ Although advances in antiepileptic drug (AED) therapy have improved the overall efficacy of seizure management, seizure response remains suboptimal in patients with cerebral AVMs. In patients with untreated AVMs managed conservatively, the 5-year risk of recurrent unprovoked seizures is estimated to be $67 \%$, whereas the 2-year seizure-freedom rate is approximately $57 \% .{ }^{19}$ In patients with incidentally discovered AVMs, the 5-year seizure risk is approximately $8 \%$. Unfortunately, uncontrolled epilepsy is associated with unemployment, psychiatric comorbidities, and significant mortality, although adequate seizure relief can diminish these effects. ${ }^{14,33}$

Stereotactic radiosurgery (SRS) is a minimally invasive management option for AVM obliteration, which reduces hemorrhage risk, patient morbidity, and mortality. ${ }^{6,26,29}$

ABBREVIATIONS AED = antiepileptic drug; ARUBA = A Randomized Trial of Unruptured Brain Arteriovenous Malformations; $A V M=$ arteriovenous malformation; IQR = interquartile range; $\mathrm{RCT}$ = randomized controlled trial; $\mathrm{SRS}$ = stereotactic radiosurgery.

SUBMITTED February 2, 2018. ACCEPTED July 19, 2018.

INCLUDE WHEN CITING Published online December 14, 2018; DOI: 10.3171/2018.7.JNS18304. 
Data from randomized controlled trials (RCTs) are limited to guide treatment decisions in cerebral AVMs. The ARUBA (A Randomized Trial of Unruptured Brain Arteriovenous Malformations) trial did not provide detailed seizure outcomes and was limited by short follow-up. ${ }^{28,36}$ There are no RCTs specifically dedicated to comparing seizure outcomes in patients with AVM treatment and those treated with conservative therapy alone.

Indications for SRS in the management of seizures have thus depended on nonrandomized case series, which have been limited by small sample size, short followup duration, and inadequate review of the AED regimen. $4,7,11,12,15,23,30,32,34$ In the current study, we describe longterm seizure outcomes in a large observational cohort of SRS-treated AVMs in patients presenting with seizure. Additionally, we characterize the temporal course of postSRS seizures and identify prognostic pre-SRS, intra-SRS, and post-SRS factors associated with long-term seizure control. Understanding factors that confer seizure relief may help in selecting AVM treatment modality, risk stratification, and the design of future RCTs.

\section{Methods \\ Patient Selection}

We conducted a retrospective review of a prospectively maintained database of 802 patients who underwent SRS for cerebral AVMs. Patients were excluded if they did not present with a history of seizure disorder $(n=595)$, if they were lost to follow-up $(n=20)$, or if a detailed seizure follow-up could not be elicited from the records $(n=23)$. Patients were also excluded if they did not meet a minimum follow-up duration of 6 months $(n=9)$. A total of 155 patients were identified who were treated with SRS for cerebral AVMs and who also initially presented with seizure disorder (Table 1). There were 75 males (48\%), and the median age at the time of SRS treatment was 35 years (interquartile range [IQR] 25-47 years). The proportion of AVMs with a Spetzler-Martin score of 3 or less was $84 \%$, and the median Pollock-Flickinger score was 1.3 (IQR $1.0-1.6)$. Treatment history included 11 prior resections (7\%). The average pre-SRS seizure frequency was either patient reported or calculated by dividing the total number of pre-SRS seizures by the time period from the date of the first pre-SRS seizure to the date of SRS. The mean pre-SRS seizure frequency was 14.2 seizures/year (95\% CI 5.4-23.1). The average period of evaluation of epilepsy prior to SRS was 11.6 months (95\% CI 3.1-73.1 months). The majority of patients presented with generalized onset seizures $(58 \%)$ and were treated with 1 AED (83\%). Preoperatively, 13 patients $(8 \%$ ) were not taking any AEDs for seizure control. Of all patients, $30(19 \%)$ presented with intracranial hemorrhage in addition to seizures, whereas the remaining 125 (81\%) presented with seizure alone.

The following study was approved by the institutional review board for retrospective analysis of human subjects.

\section{Data Collection}

For each patient we collected pre-SRS and post-SRS seizure information, including the length of the seizure disorder, seizure frequency, AED regimen, and seizure type (focal onset with or without awareness, or generalized onset). AVM characteristics collected included location, size, presence of a venous varix, coexistence of an aneurysm, and location of draining veins. AVMs located between two lobes were classified dually in both location categories. Additionally, AVMs were classified via Pollock-Flickinger and Spetzler-Martin scores. Long-term clinical follow-up information was collected via a combination of telephone conversations, emails, and in-person clinic visits. This information was supplemented with documentation from other healthcare providers involved in the patient's care. For long-term seizure outcomes, we evaluated the number of patients achieving seizure-free status, the seizure control rate, and the number of patients successfully weaned off their medication. For patients undergoing multiple SRS treatments, the radiosurgical course (preoperative, postoperative) was defined based on the initial operation. Seizurefree status was achieved if patients reported no seizure episodes for the preceding 6 months. Seizure control rate was defined as the proportion of patients reaching seizure-free status or with $>50 \%$ improvement in seizure frequency after SRS treatment as compared to their pre-SRS status. Post-SRS seizure frequency was calculated by dividing the number of post-SRS seizures by the follow-up duration.

Additionally, we classified post-SRS seizures by using a modified Engel seizure classification scale in patients with at least 2 years of clinical follow-up as a secondary outcome. ${ }^{9,30}$ Here, patients were classified into 1 of 5 groups: absence of all seizures for 1 year (Engel IA); absence of disabling seizures for 1 year (Engel IB); disabling seizures occurring rarely over 2 years (Engel II); worthwhile improvement, but for less than 2 years (Engel III); or no worthwhile improvement in seizure frequency (Engel IV). Engel scores were calculated as a secondary measure because 2 years of follow-up data are needed to accurately assess Engel classes II and III. Rather than lessen the statistical significance of our results by excluding all patients with inadequate follow-up, we used the Engel scores to validate our initial classification method in a subgroup of patients with more than 2 years of follow-up.

\section{Radiosurgery Technique}

Our approach for SRS treatment of cerebral AVMs has been previously described in detail. ${ }^{21}$ We used biplanar stereotactic angiography and MRI to define the AVM nidal target volume. Of note, radiosurgery planning with MRI supplementation was only used in select cases prior to $1991 .^{27}$ Throughout the study time frame, patients were treated with a Model U, B, C, or 4-C Leksell Gamma Knife (Elekta Inc.). The median target volume was 5.4 $\mathrm{cm}^{3}$ (IQR 2.9-8.3 $\mathrm{cm}^{3}$ ) and the median 12-Gy volume was $10.8 \mathrm{~cm}^{3}$ (IQR $6.5-14.9 \mathrm{~cm}^{3}$ ). The median maximal dose was $36 \mathrm{~Gy}$ (IQR 32-40 Gy) and the median marginal dose was 20 Gy (IQR 17-20 Gy).

\section{Follow-Up Studies}

Follow-up MRI was requested at 6-, 12-, 24-, 36-, 60-, and 84-month intervals after SRS. If any patient developed new-onset neurological deficits in the interim period, CT or MRI sequences were obtained to assess for 
any developing intracranial hemorrhage or adverse radiation effects. Adverse radiation effects included any cyst, edema, or necrosis found on post-SRS imaging studies. MRI studies obtained at 36 months were used to evaluate for AVM obliteration, and patients were subsequently referred for cerebral angiography if no residual nidus could be detected. AVM obliteration was defined as an undetectable nidus in the absence of early draining veins on angiography or MRI. ${ }^{20}$

\section{Risk Factor Analysis}

We estimated the time to seizure-free status by using Kaplan-Meier survival curves, with the date of the last seizure relative to SRS treatment serving as the event marker. Differences in seizure frequency at baseline, 1-year, 2-year, 3-year, and 4-year time points were measured via a Kruskal-Wallis H-test with a Tukey's honestly significant difference post hoc analysis. To assess the effect of SRS on medication-free status, we used a chi-square test to compare between the frequency of baseline and long-term medication-free status. We then performed a univariate risk factor analysis to evaluate for any preoperative, intraoperative, and post-SRS factors significantly associated with seizure-free status (dependent, categorical variable). Differences in the frequencies of categorical variables between patients with and without seizure-free status were evaluated using a chi-square test. A Fisher exact test was used instead if any of the counts within the contingency table was $<5$. Continuous variables were initially assessed for normality by using a Kolmogorov-Smirnov test. Associations between independent continuous variables on seizure-free status were assessed using a 2-tailed Student t-test in cases of normality or a Wilcoxon rank-sum test for nonnormal data. Factors significantly associated with seizure-free status were selected for inclusion in the multivariate logistical regression analysis, which was used to assess for independent predictors of seizure-free status.

\section{Sensitivity and Subgroup Analyses}

To evaluate the sensitivity of our risk factor analysis, we performed multivariate logistical regression in a subgroup of 128 patients with $>2$ years of clinical follow-up by using the modified Engel classification scale. Additionally, we performed a multivariate risk factor analysis in a subgroup of 71 patients achieving seizure freedom for $>$ 2 years to evaluate for factors associated with continued AED usage. Statistical significance was set at $\mathrm{p}<0.05$. Continuous variables are reported as median (IQR), unless otherwise indicated. All statistical analyses were conducted with IBM SPSS version 25 (IBM Corp.) and MATLAB 2017a (MathWorks).

\section{Results \\ AVM Outcomes}

AVM obliteration was achieved in $67 \%$ of all patients at a median of 36 months (IQR 24-62 months). In patients with at least 3 years of follow-up imaging $(n=120)$, the AVM obliteration rate was $78 \%$. Thirty-one patients (20\%) underwent repeat SRS due to incomplete AVM obliteration and, of these, 55\% (17/31 patients) achieved
TABLE 1. Characteristics of 155 patients with cerebral AVMs

\begin{tabular}{|c|c|}
\hline Variable & Value \\
\hline Median age, yrs (IQR) & $35(25-47)$ \\
\hline Male sex & $75(48 \%)$ \\
\hline \multicolumn{2}{|l|}{ Spetzler score } \\
\hline 1 & $7(5 \%)$ \\
\hline 2 & $52(34 \%)$ \\
\hline 3 & $69(45 \%)$ \\
\hline $4-6$ & $27(17 \%)$ \\
\hline Median Pollock-Flickinger score (IQR) & $1.3(1.0-1.6)$ \\
\hline \multicolumn{2}{|l|}{ AVM location } \\
\hline Frontal & $50(32 \%)$ \\
\hline Parietal & $52(34 \%)$ \\
\hline Temporal & $54(35 \%)$ \\
\hline Occipital & $19(12 \%)$ \\
\hline Subcortical & $9(6 \%)$ \\
\hline Corpus callosum & $2(1 \%)$ \\
\hline Cerebellum & $1(1 \%)$ \\
\hline Multiple AVMs & $4(3 \%)$ \\
\hline Hemorrhage & $30(19 \%)$ \\
\hline Prior resection & $11(7 \%)$ \\
\hline Median vol, $\mathrm{cm}^{3}(\mathrm{IQR})$ & $5.4(2.9-8.3)$ \\
\hline \multicolumn{2}{|l|}{ Seizure type* } \\
\hline Generalized onset & $90(58 \%)$ \\
\hline Focal awareness & $43(28 \%)$ \\
\hline Focal impaired awareness & $24(15 \%)$ \\
\hline \multicolumn{2}{|l|}{ No. of AEDs } \\
\hline 0 & $13(8 \%)$ \\
\hline 1 & $129(83 \%)$ \\
\hline 2 & $10(6 \%)$ \\
\hline 3 & $3(2 \%)$ \\
\hline Average seizures/yr (95\% Cl) & $14.2(5.4-23.1)$ \\
\hline Length of seizure disorder, mos $(95 \% \mathrm{Cl})$ & $11.6(3.1-73.1)$ \\
\hline
\end{tabular}

Values are presented as the number of patients (\%), unless otherwise noted.

* Some patients had more than one seizure type.

obliteration within a median of 3 years (IQR 2.4-4.1) after the second procedure. SRS-induced edema, necrosis, and adverse radiation effects developed in $18(12 \%), 4(3 \%)$, and $20(13 \%)$ of the patients, respectively, as demonstrated on follow-up MRI. Seven patients (5\%) experienced AVM hemorrhage post-SRS, of whom 2 patients required surgical evacuation. Two patients (1\%) developed a post-SRS cyst, of whom 1 patient $(0.6 \%)$ required surgical intervention.

\section{Post-SRS Seizure Status}

The median length of follow-up was 59 months (range 6-295 months) and the mean follow-up was 85 months (range 73-96 months). A total of 96 patients (62\%) had at least 1 post-SRS seizure. Seizure-free status was achieved in 108 patients (70\%), of whom $27 \%$ (29/108) were weaned off AEDs. An additional 23 patients (15\%) reported improved seizure frequency compared to their pre-SRS sta- 
TABLE 2. Long-term seizure and radiographic outcomes

\begin{tabular}{lc}
\hline \multicolumn{1}{c}{ Outcome } & Value \\
\hline Mean follow-up, mos (range) & $85(73-96)$ \\
\hline Seizure free at follow-up & $108(70 \%)$ \\
\hline Patients w/ seizure control ${ }^{*}$ & $131(85 \%)$ \\
\hline No. of AEDs & $28(18 \%)$ \\
\hline 0 & $89(57 \%)$ \\
\hline 1 & $23(15 \%)$ \\
\hline 2 & $4(3 \%)$ \\
\hline 3 & $10(6 \%)$ \\
\hline Unknown & $9.3(0.0-21.6)$ \\
\hline Mean seizures/yr (95\% CI) & $0.6(0.0-1.3)$ \\
\hline 1 yr & $0.2(0.1-0.4)$ \\
\hline 2 yrs & $0.1(0.0-0.2)$ \\
\hline 3 yrs & $31(20 \%)$ \\
\hline 4 yrs & $90(58 \%)$ \\
\hline Repeat SRS & $80(67 \%)$ \\
\hline AVM obliteration, initial SRS & $103(67 \%)$ \\
\hline$>3$ yrs of follow-up, $n=120$ & $93(78 \%)$ \\
\hline AVM obliteration, including repeat SRS & $20(13 \%)$ \\
\hline$>3$ yrs of follow-up, $n=120$ & $36(24-62)$ \\
\hline Time to obliteration, mos (IQR) & $18 \%)$ \\
\hline Edema & $2(1 \%)$ \\
\hline Necrosis & \\
\hline Cyst & \\
\hline Values are presented as the number of patients (\%), unless otherwise noted. \\
seizure control was defined as improved seizure rate compared to pre-SRS \\
\hline seizure rate.
\end{tabular}

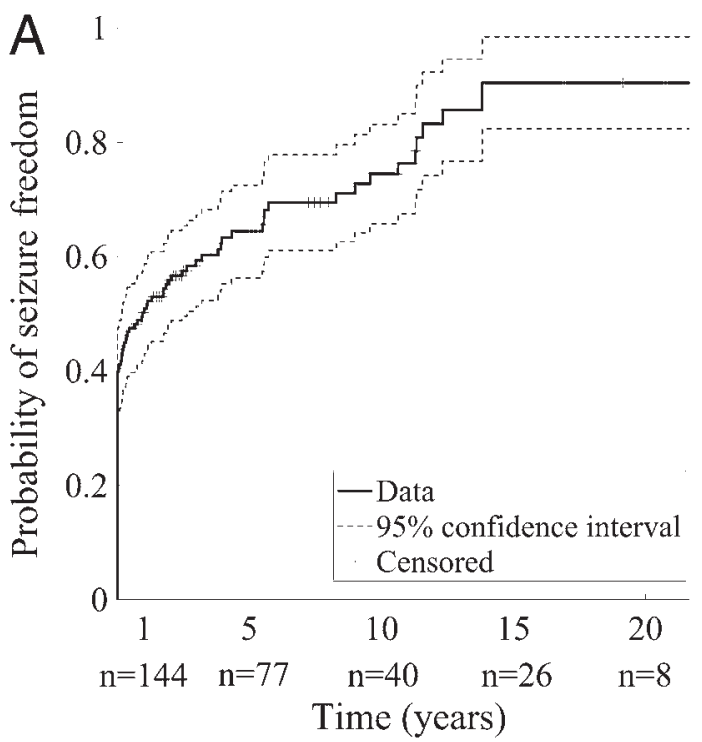

tus (Table 2). The median time to seizure-free status was estimated to be 12 months (95\% CI 0-27 months) as evaluated via Kaplan-Meier survival analysis (Fig. 1). The survival curve demonstrates that the probability of achieving seizure freedom improved with increasing follow-up duration until stabilizing at $90 \%$ at 165 months (14 years). The mean seizure frequencies at 1-, 2-, 3-, and 4-year post-SRS time points were estimated to be 9.3 (IQR 0.0-21.6), 0.6 (IQR 0.0-1.3), 0.2 (IQR 0.1-0.4), and 0.1 (IQR 0.0-0.2) episodes/year, respectively (Fig. 2). As evaluated by a Tukey's honestly significant difference post hoc analysis, the differences between pre-SRS seizure frequency and that of all post-SRS time points were found to be highly significant $(\mathrm{p}<0.00001)$. Additionally, a significant reduction in seizure frequency was noted between years 1 and $4(\mathrm{p}=0.038)$.

\section{Long-Term AED Usage}

The proportion of patients successfully weaned off all AEDs was $18 \%$ (28/155 patients). This was observed to be significantly higher than the proportion of patients without AED therapy preoperatively ( $\mathrm{p}=0.013$, chi-square test). All patients weaned off AEDs had also achieved seizurefree status (Fig. 3). The median time to medication freedom was 3.5 years (IQR 1.1-4.9) after SRS, and 2.9 years (IQR 1.1-4.9) from the last documented seizure. For a variety of reasons, 47 patients (30\%) were maintained on at least 1 AED long term, despite being seizure free for $>2$ years.

In a subgroup analysis of patients with $>2$ years of seizure freedom, we observed that patients with cortical AVM locations were more likely to be maintained on AEDs ( $p=0.028$, chi-square test). This effect was potentiated in patients with unobliterated cortical AVMs ( $\mathrm{p}=$ 0.035, chi-square test) (Fig. 4). However, AVM obliteration alone was not associated with the number of AEDs ( $p=$ 0.317, chi-square test). Other factors including age, length

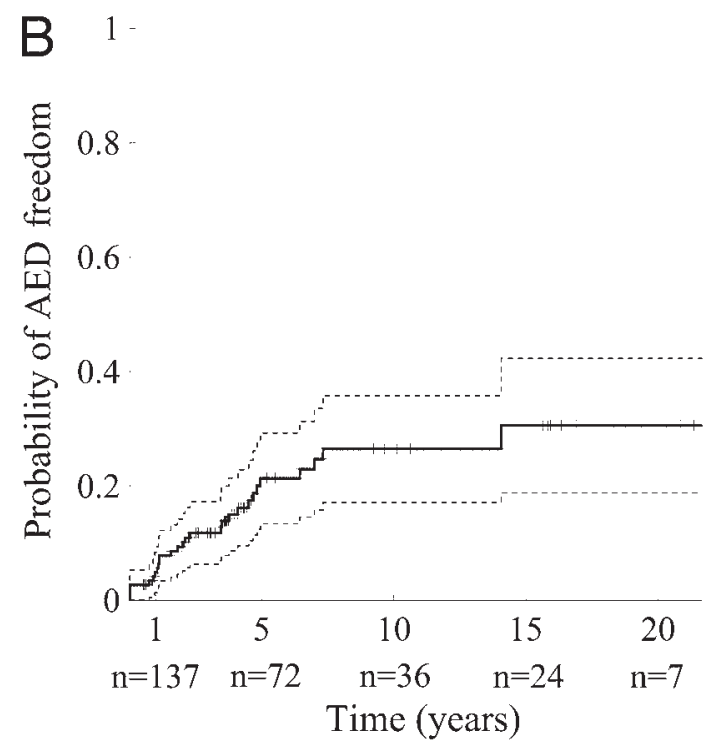

FIG. 1. Kaplan-Meier estimation for the proportion of population reaching seizure-free status (A) and medication freedom (B) with increasing follow-up. The number of patients reaching each major follow-up length is denoted below the x-axis. 


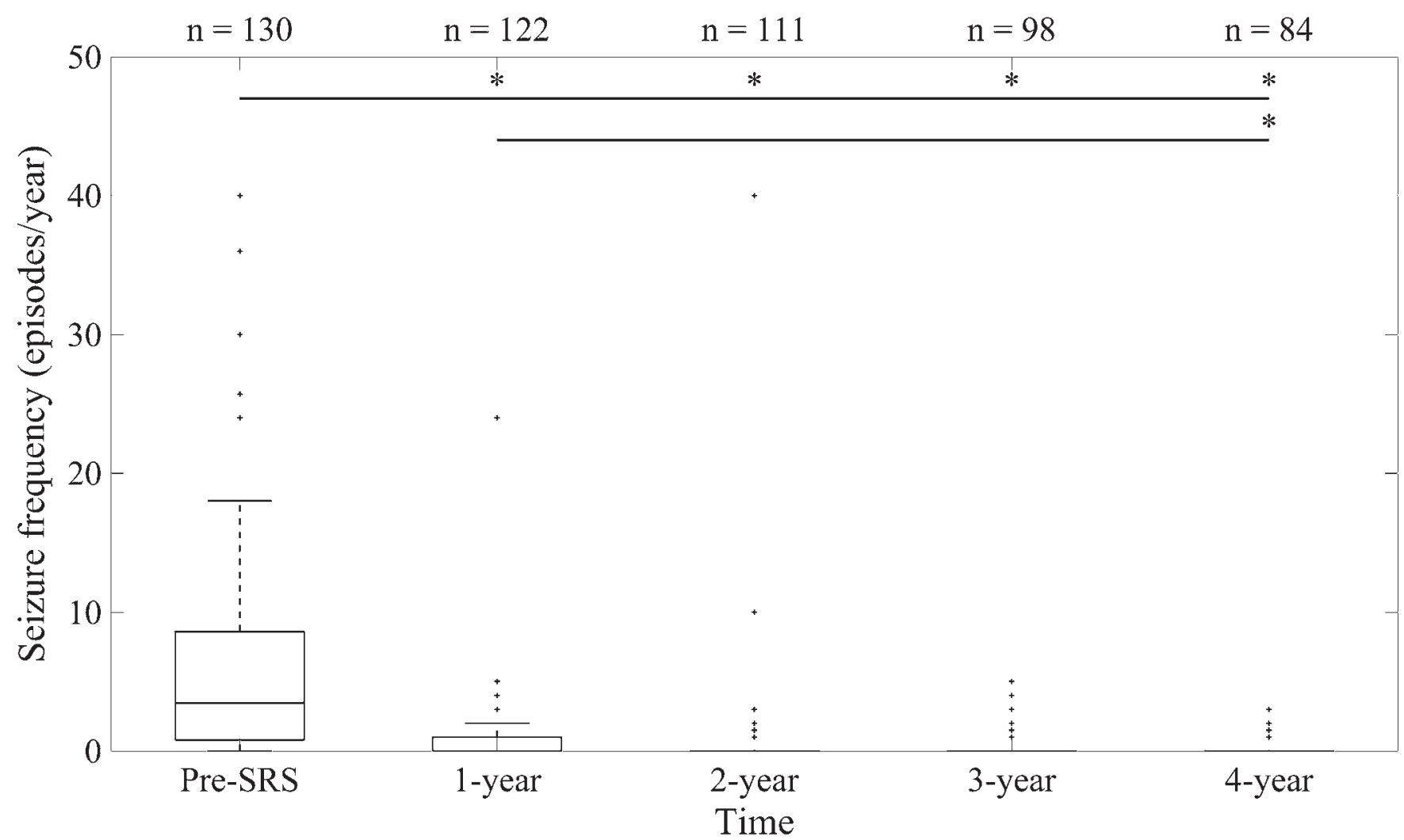

FIG. 2. The frequency of seizures preoperatively and annually for up to 4 years postoperatively. The plus signs indicate outliers in the data. Statistical significance is indicated between the leftmost portion of the horizontal lines at the top of the graph and at each of the asterisks. Not shown are seizure frequencies $>50$ episodes/year.

of seizure disorder, pre-SRS seizure frequency, presence of venous varix, superficial venous drainage, AVM multiplicity, and radiographic edema were not associated with the number of post-SRS AEDs.

\section{Risk Factors for Refractory Seizures}

We performed a univariate risk factor analysis to evaluate for any differences in pre-SRS, radiosurgical parameters, or post-SRS factors between patients with and with-

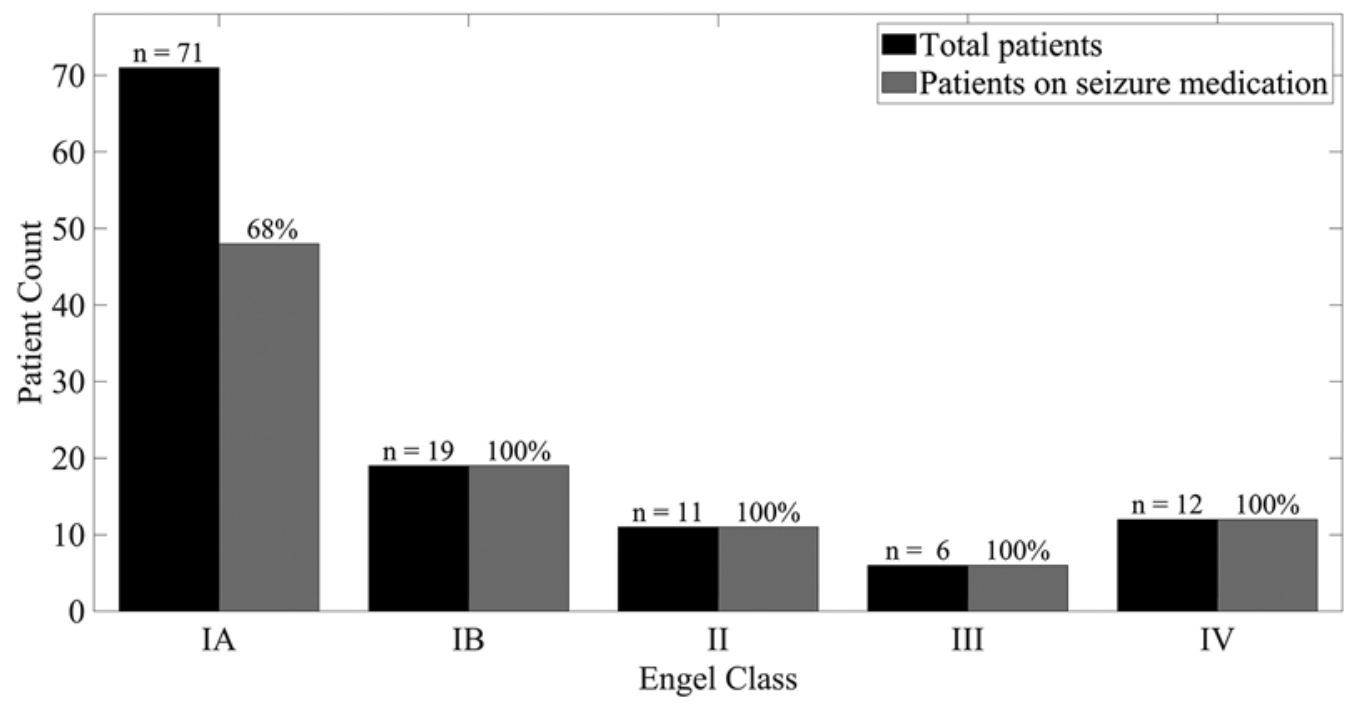

FIG. 3. Post-SRS Engel seizure classification and AED therapy of 128 patients with SRS-treated AVMs and $>2$ years of clinical follow-up ( 9 patients either had missing data to make an Engel classification [3 patients] or were missing postoperative AED data [6 patients]). Percentages are displayed as a proportion of the total patient count within each Engel classification. 


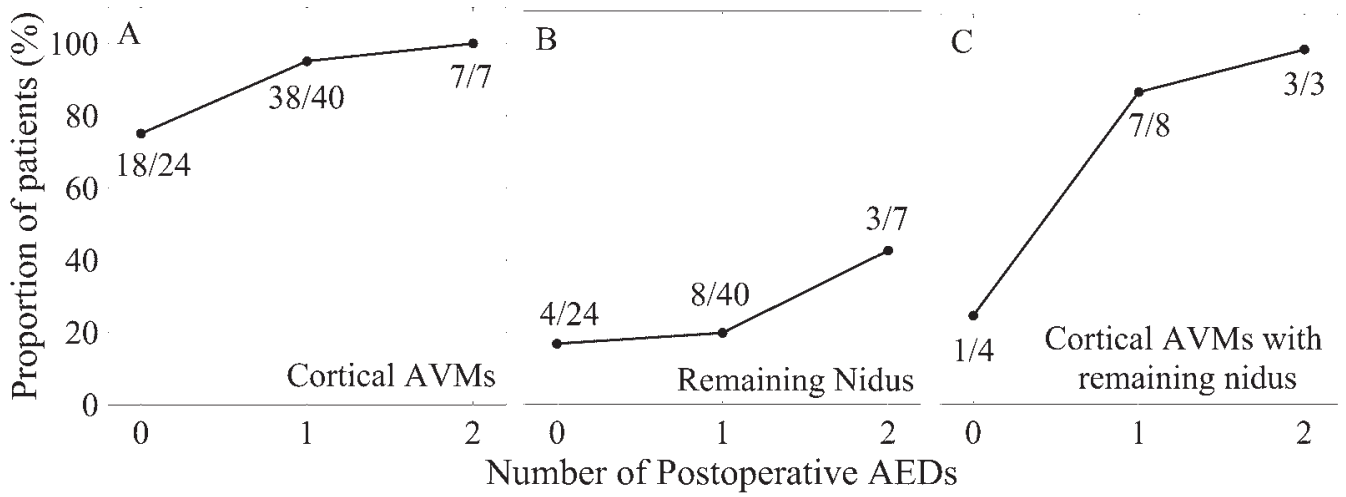

FIG. 4. Post-SRS AED regimen in patients with $>2$ years of seizure freedom as a function of AVM location (A), obliteration status (B), and location of unobliterated AVMs (C). Patients with cortical AVMs ( $p=0.028$, chi-square test) and unobliterated cortical AVMs ( $p=0.035$, chi-square test) were maintained on an increasing number of AEDs. AVM obliteration alone was not associated with AED count ( $p=0.317$, chi-square test).

out seizures at long-term follow-up (Table 3). Left-sided AVMs $(p=0.030)$, superficial venous drainage $(p=0.014)$, and focal impaired awareness seizures $(\mathrm{p}=0.031)$ were all negatively associated with seizure-free status. Other factors such as age, Spetzler-Martin score, hemorrhage (preand post-SRS), prior resection, radiation dosing parameters, and AVM obliteration were found to be nonsignificant correlates. Seizure freedom was not influenced by AVMs located in frontal $(\mathrm{p}=0.981)$, parietal $(\mathrm{p}=0.197)$, temporal $(p=0.677)$, or occipital lobes $(p=0.491)$; presence of a venous varix $(p=0.172)$; or aneurysm coexistence $(p=$ 0.345) (not shown). In 3 patients, the development of SRSinduced edema was temporally associated with seizure exacerbation. Although these patients had persistent seizures at long-term follow-up, their seizure frequency had greatly improved and all were ultimately Engel class IA or IB.

Collectively, post-SRS complications such as cyst formation $(\mathrm{p}=0.519)$, edema $(\mathrm{p}=0.982)$, necrosis $(\mathrm{p}=1.000)$, and adverse radiation effects $(p=0.820)$ were not statistically associated with seizure outcomes. We then used AVM side, superficial venous drainage, and focal impaired awareness seizure type (also known as complex partial seizures) as inputs into the multivariate logistic regression model (Table 4). On multivariate logistic regression, focal impaired awareness seizure type and superficial venous drainage were significantly associated with decreased odds ratios for seizure-free status at last follow-up (OR 0.36 [95\% CI 0.14-0.92] for focal impaired awareness seizures; OR 0.41 [95\% CI 0.18-0.93] for superficial venous drainage). Left-sided AVMs were not statistically associated with long-term seizure outcome (OR 0.48, 95\% CI 0.22-1.09).

\section{Sensitivity Analysis}

We evaluated the sensitivity of our results by using a modified Engel seizure classification scale in 128 patients who had at least 2 years of follow-up. Here, 90 patients $(70 \%)$ achieved Engel IA $(n=71)$ or IB $(n=19)$ status (Fig. 3). Of the 71 patients achieving Engel IA status, 32\% were also weaned off all AED therapy. In classes IB and higher, $100 \%$ of patients were still on active AED therapy. We performed a multivariate logistic regression analysis to evaluate the effects of left-sided AVMs, superficial ve- nous drainage, and focal impaired awareness seizure type on the odds ratio for Engel class I seizure outcome. Focal impaired awareness seizure type was associated with a decreased likelihood of achieving Engel class I (OR 0.33, 95\% CI 0.12-0.92). However, superficial venous drainage (OR 0.67, 95\% CI 0.27-1.65) and AVM side (OR 0.95, 95\% CI 0.39-2.32) were not associated with Engel class I outcomes.

\section{Discussion}

This study describes seizure outcomes in a large cohort of patients with AVM who underwent SRS. The main finding of this study is that SRS provides long-term seizure control in patients harboring cerebral AVMs. The proportion of patients achieving seizure freedom, the frequency of episodes, and the rate of medication freedom all improved with increasing clinical follow-up. Superficial venous drainage and pre-SRS focal impaired awareness seizure type were significantly associated with persistent seizures, although the presence of superficial draining veins did not predict seizure response in patients with $>2$ years of clinical follow-up. Of note, AVM obliteration and radiographic parameters were not associated with long-term seizure outcomes.

In the present study, the overall rate of seizure freedom after SRS was 70\% at a mean follow-up of 85 months (range 73-96 months). Our results concur with seizure rates reported by Baranoski et al., ${ }^{1}$ who estimated a seizure-freedom rate of $63 \%$ (range 55\%-70\%) after a mean follow-up of $44.4 \pm 21.6$ months. The meta-analysis by Chen et al. estimated that seizure freedom after SRS is approximately $53 \%$ (range $40 \%-67 \%$ ) at a mean follow-up of 46 months (range 14-93 months), ${ }^{4}$ which is somewhat lower than that observed in our study. The difference may be due to the long-term follow-up achieved in the present study, because the antiepileptic effects of SRS are thought to be time dependent.

Bowden et al. studied 87 patients who underwent SRS for AVMs of the sylvian fissure. ${ }^{3}$ Of these, 36 patients experienced seizures before SRS. The risk for pre-SRS seizures was lower for younger patients and for patients with 
TABLE 3. Univariate effects on long-term seizure outcomes

\begin{tabular}{|c|c|c|c|}
\hline \multirow[b]{2}{*}{ Variable } & \multicolumn{2}{|c|}{ Seizure at Follow-Up } & \multirow[b]{2}{*}{ p Value } \\
\hline & No, $n=108$ & Yes, $n=47$ & \\
\hline \multicolumn{4}{|l|}{ Pre-SRS factors } \\
\hline Age, yrs (IQR) & $34(24-46)$ & $36(29-48)$ & 0.217 \\
\hline Male sex & $53(49 \%)$ & $22(47 \%)$ & 0.755 \\
\hline Lt side & $58(54 \%)$ & $34(72 \%)$ & $0.030^{*}$ \\
\hline AVM location, subcortical† & $8(7 \%)$ & $1(2 \%)$ & 0.280 \\
\hline Superficial drainage & $52(48 \%)$ & $31(66 \%)$ & $0.014^{*}$ \\
\hline Spetzler score & & & 0.601 \\
\hline $1-3$ & $88(81 \%)$ & $40(85 \%)$ & \\
\hline $4-6$ & $20(19 \%)$ & $7(15 \%)$ & \\
\hline Pollock-Flickinger score (IQR) & $1.3(1.0-1.6)$ & $1.4(1.1-1.7)$ & 0.179 \\
\hline Multiple AVMs & $4(4 \%)$ & $0(0 \%)$ & 0.315 \\
\hline Hemorrhage & $22(20 \%)$ & $8(17 \%)$ & 0.840 \\
\hline Prior resection & $9(8 \%)$ & $2(4 \%)$ & 0.506 \\
\hline Diagnosis to op time, mos (IQR) & $10(3-59)$ & $23(4-94)$ & 0.198 \\
\hline \multicolumn{4}{|l|}{ Seizure type } \\
\hline Generalized onset & $64(59 \%)$ & $26(55 \%)$ & 0.385 \\
\hline Focal awareness & $29(27 \%)$ & $14(30 \%)$ & 0.848 \\
\hline Focal impaired awareness & $13(12 \%)$ & $12(26 \%)$ & $0.031^{*}$ \\
\hline Mean seizures/yr $(95 \% \mathrm{Cl})$ & $9.9(0.6-19.2)$ & $23.1(1.8-44.3)$ & 0.682 \\
\hline Length of seizure disorder, mos (IQR) & $10.3(2.7-61.4)$ & $14.9(3.7-98.0)$ & 0.204 \\
\hline Vol, $\mathrm{cm}^{3}(\mathrm{IQR})$ & $5.3(2.9-8.3)$ & $5.7(3.2-8.6)$ & 0.318 \\
\hline \multicolumn{4}{|l|}{ Radiosurgical factors (IQR) } \\
\hline$\%$ isodose & $50.0(50.0-50.0)$ & $50.0(50.0-50.0)$ & 0.219 \\
\hline Maximal dose, Gy & $36.0(32.7-40.0)$ & $36.0(32.0-40.0)$ & 0.562 \\
\hline Marginal dose, Gy & $20.0(17.5-20.0)$ & $19.0(17.0-20.0)$ & 0.285 \\
\hline 12-Gy vol, $\mathrm{cm}^{3}$ & $10.2(6.1-14.8)$ & $12.2(6.9-15.3)$ & 0.163 \\
\hline \multicolumn{4}{|l|}{ Post-SRS factors } \\
\hline AVM obliteration & $75(70 \%)$ & $28(60 \%)$ & 0.202 \\
\hline Time to obliteration, mos (IQR) & $37(24-59)$ & $31(22-62)$ & 0.619 \\
\hline Repeat SRS & $20(19 \%)$ & $11(23 \%)$ & 0.576 \\
\hline Edema & $12(11 \%)$ & $6(13 \%)$ & 0.982 \\
\hline Necrosis & $3(3 \%)$ & $1(2 \%)$ & 1.000 \\
\hline Cyst & $1(2 \%)$ & $1(2 \%)$ & 0.519 \\
\hline Adverse radiation effect & $13(12 \%)$ & $7(15 \%)$ & 0.820 \\
\hline Follow-up time, mos (IQR) & $61(32-150)$ & $49(31-92)$ & 0.686 \\
\hline
\end{tabular}

Values are presented as the number of patients (\%), unless otherwise noted.

* Indicates statistical significance $(p<0.05)$.

† Thalamus or basal ganglia.

a history of previous intracranial hemorrhage. At the last follow-up, 19 patients (53\%) had achieved Engel class I (free of disabling seizures), 4 (11\%) had class II (rare disabling seizures), and 2 (6\%) had class III (significant improvement) status. There was no improvement (class IV) in 11 patients $(31 \%)$. After SRS, clinically significant seizure reduction of at least 1 Engel class was documented for 17 $(47 \%)$ of the 36 patients. The median time to seizure reduction was 20 months (range 1-129 months). The rates of seizure improvement were $29 \%$ at 3 years, $36 \%$ at 5 years, $50 \%$ at 10 years, and $60 \%$ at 15 years. In that study, the rate of improved seizure control was higher for patients with
TABLE 4. Effects on odds ratio for seizure freedom

\begin{tabular}{lccccc}
\hline & \multirow{2}{*}{$\begin{array}{c}\text { OR for } \\
\text { Variable }\end{array}$} & \multicolumn{2}{c}{$95 \% \mathrm{Cl}$} & \\
\cline { 3 - 5 } & Seizure-Free & & \\
Status & Lower & Upper & Value \\
\hline Lt side & 0.48 & 0.22 & 1.09 & 0.080 \\
\hline Focal impaired awareness & 0.36 & 0.14 & 0.92 & $0.033^{*}$ \\
\hline Superficial venous drainage & 0.41 & 0.18 & 0.93 & $0.033^{*}$ \\
\hline * Indicates statistical significance $(p<0.05)$. & & &
\end{tabular}


incomplete obliteration $(\mathrm{p}=0.042)$, lower among patients with a history of prior hemorrhage, and higher for patients with small nidus diameter $(<2 \mathrm{~cm})$. In comparison to the current study, seizures may be more difficult to control and may have different prognosticating factors (obliteration, hemorrhage, and nidus size) in patients with sylvian fissure seizures.

Of note, our results indicate that the proportion of patients reaching freedom from maintenance AED therapy $(18 \%)$ was lower than that reported in previous studies. ${ }^{7,30,39}$ In general, our approach has been to attempt to wean patients off AEDs after 2 years of seizure freedom. ${ }^{4}$ Nonetheless, we found that many patients were maintained on 1 AED despite AVM obliteration and seizure freedom for $>$ 2 years. In that regard, we observed that a high proportion (96\%) of patients harboring cortical AVMs were on a continued AED regimen, despite long-term seizure freedom (Fig. 4), and this finding was potentiated in unobliterated cortical AVMs. It is possible that patients were continued on AEDs by their physicians as a precautionary measure out of concern for AVMs located in epileptogenic areas, especially if medications were well tolerated. It is also possible that many patients felt comfortable on medication and did not want to risk a seizure during the weaning-off period, an event which could have resulted in loss of driving privileges and often of employment. For patients successfully weaned off medications, the median time to medication freedom was 3.5 years from SRS, which was consistent with that reported by Yang et al. ${ }^{39}$

There have been few data from RCTs to demonstrate seizure benefit of interventional treatment compared to conservative management of brain AVMs. A recent meta-analysis concluded that microsurgical resection $(78 \%$ seizure freedom) offered superior seizure control as compared to SRS (63\% seizure freedom) and endovascular embolization (49\% seizure freedom). ${ }^{1}$ However, the highest rate of seizure freedom was observed in patients who had an AVM that was obliterated after SRS (85\% seizure freedom). Additionally, the risk of new-onset seizures was lowest in the SRS group compared to that of the surgical and endovascular groups. Wang et al. further demonstrated that new-onset seizures were more frequent in patients undergoing microsurgical resection compared to those receiving SRS. ${ }^{37}$

Whether SRS offers superior seizure control compared to medical therapy alone remains unclear. The ARUBA trial showed that the number of seizures was similar between patients receiving interventional or medical therapy alone, regardless of seizure semiology. ${ }^{28}$ However, the study did not evaluate detailed seizure outcomes and was limited by short follow-up. In a population-based observational cohort of 229 patients, Josephson et al. evaluated seizure outcomes of AVMs treated by excision, embolization, and SRS as compared to those of patients treated with conservative management alone. ${ }^{19}$ The study concluded that there were no significant differences in the 5-year risk of recurrent unprovoked seizures and 2-year seizure-free rates between AVM treatment options and conservative management. Although no significant differences were observed, the authors noted that patients in the intervention group had higher baseline seizure frequency and more temporal lobe AVMs, and thus may have had a higher propensity for long-term refractory seizures. Neither of the aforementioned studies evaluated the effects of SRS alone.

Previous studies have had mixed results regarding the effects of AVM obliteration on long-term seizure relief. A recent meta-analysis of 19 case series and 997 patients with AVM concluded that AVM obliteration confers superior seizure control compared to that in patients with incomplete AVM obliteration. ${ }^{4}$ SRS-induced AVM occlusion probably has antiepileptic effects by reducing the vascular steal phenomenon, thus restoring normal blood flow to the surrounding parenchyma. However, two studies published after the one by Chen et al. ${ }^{4}$ that included 151 total patients demonstrated that AVM obliteration does not necessarily predict seizure outcomes. ${ }^{7,30}$ In our study, we observed that seizure remission was not significantly associated with AVM obliteration, suggesting that SRS provides at least some seizure control via radiation to the surrounding tissue. , $^{42,15}$ Although not well understood, it has been suggested that perinidal radiation provides seizure relief through multiple mechanisms, including inhibition of protein synthesis, neuromodulatory effects on local inhibitory and excitatory neurons, perinidal gliotic capsule formation, dendritic sprouting, and cortical reorganization. .,4,31,38 $^{2}$

Additionally, the role of superficial venous drainage has been suggested to increase seizure risk, ${ }^{11,18}$ which was also observed in our study. In these patients, epileptogenic activity may be driven by mass effect on surrounding cortical tissue via high-flow pulsating draining veins or through dural venous lake formation. ${ }^{11,24}$ Superficial draining veins may also decrease the seizure threshold via ischemia to the surrounding brain tissue as a result of venous congestion. ${ }^{10,24}$ A recent radiographic study of 20 patients reported that all patients with AVMs who presented with seizures had diminished perinidal cerebrovascular reserve due to impaired cerebral autoregulation. ${ }^{10}$ On subsequent angiography, the authors observed restricted venous outflow in patients with seizure, suggesting that venous congestion increases seizure risk via compromised perinidal cerebrovascular reactivity. Of note, we observed that the effects of superficial venous drainage on persistent seizures was no longer significant once patients with short-term follow-up ( $<2$ years) were excluded. Our results suggest that local effects of venous drainage may play a role in short-term seizure propagation, but the antiepileptic effects of SRS predominate in the long term.

It is unclear, however, why patients with focal impaired awareness seizure type were more likely to experience long-term persistent seizures. Our study agrees with prior reports, which have shown that patients with focal aware and motor generalized onset seizures are more likely to experience remission with AVM treatment compared to focal impaired awareness seizure type. ${ }^{16,17,30,32}$ However, other studies have been unable to demonstrate such an effect. ${ }^{4,8,23}$ Other factors related to poor seizure outcomes that were not observed in our study include high pre-SRS seizure frequency, ${ }^{23,32,39}$ long seizure history, ${ }^{23}$ low SRS margin dose, ${ }^{22}$ AVM hemorrhage,,$^{12}$ large AVM size, ${ }^{32}$ and frontal location. ${ }^{30}$

The results of our study are probably affected by several methodological factors. One weakness of our study is 
that seizure activity was diagnosed clinically in most cases and was not always localized with electroencephalography monitoring. We cannot exclude the possibility that some of the patient-reported clinical events may have been pseudoseizures. One prior study has shown that AVMs are often associated with distal extranidal epileptic foci and that excising these foci can result in superior seizure control. ${ }^{13,35,40}$ Future prospective studies should thus correlate clinical seizures with neurophysiological data in order to identify and target secondary epileptogenic structures. In our analysis of pre-SRS seizure frequency, it should be noted that the mean seizure disorder duration was $<1$ year (11.6 months). This could suggest a clustering of seizures around the time of SRS, which may have influenced our post-SRS seizure control rate. Additionally, we excluded patients who did not present with a history of seizure disorder, and thus we could not evaluate the rate of de novo seizures after SRS. Another weakness of our study is that we did not assess the number of patients who experienced seizure relapse after an attempted AED wean, and it would be valuable to assess this in future studies. Finally, these data are a retrospective review of patient outcomes from a single institution. Accordingly, it may be difficult to generalize our results to other institutions, which may have varying experience with SRS treatment of AVMs. Further research, including randomized and blinded multicenter studies of AVM-associated seizures, may further inform risk stratification and indications for treatment.

\section{Conclusions}

Patients undergoing SRS for cerebral AVMs experience increased likelihood of seizure freedom, decreased seizure frequency, and long-term freedom from medication. Antiepileptic effects of SRS may occur independently of AVM obliteration. Focal impaired awareness seizure type may represent a risk factor in long-term seizure control. Superficial venous drainage may propagate overall seizure risk, although this effect may be diminished over long-term follow-up.

\section{References}

1. Baranoski JF, Grant RA, Hirsch LJ, Visintainer P, Gerrard JL, Günel M, et al: Seizure control for intracranial arteriovenous malformations is directly related to treatment modality: a meta-analysis. J Neurointerv Surg 6:684-690, 2014

2. Barcia-Salorio JL: Radiosurgery in epilepsy and neuronal plasticity. Adv Neurol 81:299-305, 1999

3. Bowden G, Kano H, Tonetti D, Niranjan A, Flickinger J, Arai Y, et al: Stereotactic radiosurgery for sylvian fissure arteriovenous malformations with emphasis on hemorrhage risks and seizure outcomes. J Neurosurg 121:637-644, 2014

4. Chen CJ, Chivukula S, Ding D, Starke RM, Lee CC, Yen CP, et al: Seizure outcomes following radiosurgery for cerebral arteriovenous malformations. Neurosurg Focus 37(3):E17, 2014

5. Crawford PM, West CR, Shaw MD, Chadwick DW: Cerebral arteriovenous malformations and epilepsy: factors in the development of epilepsy. Epilepsia 27:270-275, 1986

6. Ding D, Yen CP, Xu Z, Starke RM, Sheehan JP: Radiosurgery for patients with unruptured intracranial arteriovenous malformations. J Neurosurg 118:958-966, 2013

7. Ditty BJ, Omar NB, Foreman PM, Miller JH, Kicielinski
KP, Fisher WS III, et al: Seizure outcomes after stereotactic radiosurgery for the treatment of cerebral arteriovenous malformations. J Neurosurg 126:845-851, 2017

8. Eisenschenk S, Gilmore RL, Friedman WA, Henchey RA: The effect of LINAC stereotactic radiosurgery on epilepsy associated with arteriovenous malformations. Stereotact Funct Neurosurg 71:51-61, 1998

9. Engel J, Van Ness P, Rasmussen T, Ojemann L: Outcome with respect to epileptic seizures, in Jerome Engel J (ed): Surgical Treatment of the Epilepsies, ed 2. New York: Raven Press, 1993, pp 609-621

10. Fierstra J, Conklin J, Krings T, Slessarev M, Han JS, Fisher $\mathrm{JA}$, et al: Impaired peri-nidal cerebrovascular reserve in seizure patients with brain arteriovenous malformations. Brain 134:100-109, 2011

11. Garcin B, Houdart E, Porcher R, Manchon E, Saint-Maurice JP, Bresson D, et al: Epileptic seizures at initial presentation in patients with brain arteriovenous malformation. Neurology 78:626-631, 2012

12. Gerszten PC, Adelson PD, Kondziolka D, Flickinger JC, Lunsford LD: Seizure outcome in children treated for arteriovenous malformations using gamma knife radiosurgery. Pediatr Neurosurg 24:139-144, 1996

13. Goldensohn ES: The relevance of secondary epileptogenesis to the treatment of epilepsy: kindling and the mirror focus. Epilepsia 25 (Suppl 2):S156-S173, 1984

14. Guekht AB, Mitrokhina TV, Lebedeva AV, Dzugaeva FK, Milchakova LE, Lokshina OB, et al: Factors influencing on quality of life in people with epilepsy. Seizure 16:128-133, 2007

15. Heikkinen ER, Konnov B, Melnikov L, Yalynych N, Zubkov YuN, Garmashov YuA, et al: Relief of epilepsy by radiosurgery of cerebral arteriovenous malformations. Stereotact Funct Neurosurg 53:157-166, 1989

16. Hoh BL, Chapman PH, Loeffler JS, Carter BS, Ogilvy CS: Results of multimodality treatment for 141 patients with brain arteriovenous malformations and seizures: factors associated with seizure incidence and seizure outcomes. Neurosurgery 51:303-311, 2002

17. Hyun SJ, Kong DS, Lee JI, Kim JS, Hong SC: Cerebral arteriovenous malformations and seizures: differential impact on the time to seizure-free state according to the treatment modalities. Acta Neurochir (Wien) 154:1003-1010, 2012

18. Jiang P, Lv X, Wu Z, Li Y, Jiang C, Yang X, et al: Characteristics of brain arteriovenous malformations presenting with seizures without acute or remote hemorrhage. Neuroradiol J 24:886-888, 2011

19. Josephson CB, Bhattacharya JJ, Counsell CE, Papanastassiou V, Ritchie V, Roberts R, et al: Seizure risk with AVM treatment or conservative management: prospective, populationbased study. Neurology 79:500-507, 2012

20. Kano H, Kondziolka D, Flickinger JC, Yang HC, Flannery TJ, Awan NR, et al: Stereotactic radiosurgery for arteriovenous malformations, part 3: outcome predictors and risks after repeat radiosurgery. J Neurosurg 116:21-32, 2012

21. Kano H, Kondziolka D, Flickinger JC, Yang HC, Park KJ, Flannery TJ, et al: Aneurysms increase the risk of rebleeding after stereotactic radiosurgery for hemorrhagic arteriovenous malformations. Stroke 43:2586-2591, 2012

22. Kida Y, Kobayashi T, Tanaka T, Mori Y, Hasegawa T, Kondoh T: Seizure control after radiosurgery on cerebral arteriovenous malformations. J Clin Neurosci 7 (Suppl 1):6-9, 2000

23. Kurita H, Kawamoto S, Suzuki I, Sasaki T, Tago M, Terahara A, et al: Control of epilepsy associated with cerebral arteriovenous malformations after radiosurgery. J Neurol Neurosurg Psychiatry 65:648-655, 1998

24. Lasjaunias P, Chiu M, ter Brugge K, Tolia A, Hurth M, Bernstein M: Neurological manifestations of intracranial dural 
arteriovenous malformations. J Neurosurg 64:724-730, 1986

25. Leblanc R, Feindel W, Ethier R: Epilepsy from cerebral arteriovenous malformations. Can J Neurol Sci 10:91-95, 1983

26. Liscák R, Vladyka V, Simonová G, Urgosík D, Novotný J Jr, Janousková L, et al: Arteriovenous malformations after Leksell gamma knife radiosurgery: rate of obliteration and complications. Neurosurgery 60:1005-1016, 2007

27. Lunsford LD, Kondziolka D, Flickinger JC, Bissonette DJ, Jungreis CA, Maitz AH, et al: Stereotactic radiosurgery for arteriovenous malformations of the brain. J Neurosurg 75:512-524, 1991

28. Mohr JP, Parides MK, Stapf C, Moquete E, Moy CS, Overbey $\mathrm{JR}$, et al: Medical management with or without interventional therapy for unruptured brain arteriovenous malformations (ARUBA): a multicentre, non-blinded, randomised trial. Lancet 383:614-621, 2014

29. Nataf F, Schlienger M, Bayram M, Ghossoub M, George B, Roux FX: Microsurgery or radiosurgery for cerebral arteriovenous malformations? A study of two paired series. Neurosurgery 61:39-50, 2007

30. Przybylowski CJ, Ding D, Starke RM, Yen CP, Quigg M, Dodson B, et al: Seizure and anticonvulsant outcomes following stereotactic radiosurgery for intracranial arteriovenous malformations. J Neurosurg 122:1299-1305, 2015

31. Quigg M, Rolston J, Barbaro NM: Radiosurgery for epilepsy: clinical experience and potential antiepileptic mechanisms. Epilepsia 53:7-15, 2012

32. Schäuble B, Cascino GD, Pollock BE, Gorman DA, Weigand S, Cohen-Gadol AA, et al: Seizure outcomes after stereotactic radiosurgery for cerebral arteriovenous malformations. Neurology 63:683-687, 2004

33. Sperling MR, Feldman H, Kinman J, Liporace JD, O'Connor MJ: Seizure control and mortality in epilepsy. Ann Neurol 46:45-50, 1999

34. Steiner L, Lindquist C, Adler JR, Torner JC, Alves W, Steiner $\mathrm{M}$ : Clinical outcome of radiosurgery for cerebral arteriovenous malformations. J Neurosurg 77:1-8, 1992

35. Sturiale CL, Puca A, Albanese A, Maira G, Colicchio G: A comment on impaired peri-nidal cerebrovascular reserve in seizure patients with brain arteriovenous malformations. Brain 135:e197-e198, 2012

36. Tonetti DA, Gross BA, Atcheson KM, Jankowitz BT, Kano $\mathrm{H}$, Monaco EA III, et al: The benefit of radiosurgery for ARUBA-eligible arteriovenous malformations: a practical analysis over an appropriate follow-up period. J Neurosurg 128:1850-1854, 2018

37. Wang JY, Yang W, Ye X, Rigamonti D, Coon AL, Tamargo RJ, et al: Impact on seizure control of surgical resection or radiosurgery for cerebral arteriovenous malformations. Neurosurgery 73:648-656, 2013

38. Yang I, Barbaro NM: Advances in the radiosurgical treatment of epilepsy. Epilepsy Curr 7:31-35, 2007

39. Yang SY, Kim DG, Chung HT, Paek SH: Radiosurgery for unruptured cerebral arteriovenous malformations: long-term seizure outcome. Neurology 78:1292-1298, 2012

40. Yeh HS, Kashiwagi S, Tew JM Jr, Berger TS: Surgical management of epilepsy associated with cerebral arteriovenous malformations. J Neurosurg 72:216-223, 1990

\section{Disclosures}

Dr. Lunsford is an AB Elekta stockholder and a consultant for Insightec, DSMB

\section{Author Contributions}

Conception and design: Niranjan, Kashkoush. Acquisition of data: Kashkoush, Kano. Analysis and interpretation of data: Niranjan, Kashkoush. Drafting the article: Kashkoush. Critically revising the article: Niranjan, Kano, Monaco, Flickinger, Lunsford. Reviewed submitted version of manuscript: all authors. Approved the final version of the manuscript on behalf of all authors: Niranjan.

\section{Correspondence}

Ajay Niranjan: University of Pittsburgh, PA.niranjana@ upmc.edu. 